\title{
Les 400 coups d'un Écolier Sacripant. Une approche du comique chez Valère Novarina
}

\section{Thierry Toulze}

\section{(2) OpenEdition}

1 Journals

Édition électronique

URL : http://journals.openedition.org/recherchestravaux/306

DOI : 10.4000/recherchestravaux.306

ISSN : 1969-6434

Éditeur

UGA Éditions/Université Grenoble Alpes

\section{Édition imprimée}

Date de publication : 15 octobre 2006

Pagination : 97-108

ISBN : 978-2-84310-099-4

ISSN : 0151-1874

\section{Référence électronique}

Thierry Toulze, «Les 400 coups d'un Écolier Sacripant. Une approche du comique chez Valère Novarina », Recherches \& Travaux [En ligne], 69 | 2006, mis en ligne le 18 décembre 2013, consulté le 07 septembre 2020. URL : http://journals.openedition.org/recherchestravaux/306 ; DOI : https:// doi.org/10.4000/recherchestravaux.306 


\section{Les 400 coups d'un Écolier Sacripant Une approche du comique chez Valère Novarina}

Chez Valère Novarina, chaque phrase ou presque nous paraît placée sous le signe de l'humour, de la farce et de la facétie, un peu comme s'il s'agissait de dérouter d'éventuelles "Brigades rhétoriques» (et autres "grammato-radotiens", "perroquateurs de concepts ", «dogmatosés ", «médiagogues » ou "orthoscénistes » toujours à l'affût du moindre écart) en procédant avec toute l'insolence d'un enfant intenable qui se réjouirait in petto de l'incompréhension totale suscitée par un fou babil néobabéliennement pluriloque, ou qui lancerait gaiement «Bisque, bisque, rage!» à la face de quiconque brûlerait de plaquer mécaniquement les tristes grilles d'une "glose de glas» cuistrement mortifere.

Crâne funambule prenant le risque de basculer complètement dans le ridicule, le "petit exégète perdu» (comme l'aurait peut-être qualifié Artaud) pourra cependant tenter d'entrer novariniennement en piste pour faire le "sacrifice comique» d'un article universitaire visant à analyser les modalités d'apparition de "l'énergie enfantine [...] ici délivrée ${ }^{2}$ ».

De fait, si l'on sait à peu près en quoi consiste la "cancritude logique» de l' «Enfant Valère», son goût de mots comme "soustractionner » ou « un animau " et son manifeste implicite pour un Bescherelle revisité, il nous semble que la dimension potache, dans une acception jarryque, de ses "actions comiques" n'a pas encore été suffisamment étudiée. C'est surtout dans Le Discours aux animaux qu'ont lieu ses 400 coups langagiers. Ne notons que "pourrir de rire», «mi-figue mi-scorpion », "[avoir] la bête vide», "faire chou-bœuf» et

I. Du nom d'un des innombrables «Enfants» de l'œuvre novarinienne. Mentionné notamment dans Le Discours aux animaux, 1987, et dans L'Animal du temps, 1993.

2. Formule du Bonhomme de Terre dans Le Jardin de reconnaissance, 1997, p. 99. Comme pour toutes les œuvres citées ici, il s'agit des éditions P.O.L. (Paris), précision qui ne sera plus donnée par la suite. 
«sauve-qui-pond» (sans oublier «avanir», «nulle-patte» et "polichier»). Cela posé, dans l'arsenal potache en question, ready-made, à-peu-près, épenthèse et suppression-adjonction ne seront certainement pas les seules armes utilisées. C'est qu'à l'instar de l'Écrivain Sacripant, le narrateur-auteur du Discours se veut essentiellement brouilleur de pistes et de cartes, semeur de trouble et porteur de confusion: "J'inscrivais nulle part en flèches vite faites des fuites indicatives vers des culs-de-sacs partout» (p. 232). Aussi bien, notre tagueur fou ${ }^{3}$ pourra à l'occasion se métamorphoser en oiseau de mauvais augure (“j'écris en bombe dans un métro: "Humanité, retourne en larve, ta marche arrière a commencé" " [p. 98]) ou se montrer carrément injurieux pour l'éventuel lectorat: "sachez que celui qui écrivit ceci n’a rien à dire mais vous emmerde», phrase adoucie (en «mais vous l'répète») dans la version scénique 4 écrite pour l'acteur André Marcon.

Toutefois, il semblerait que le terrorisme en question présente d'abord des caractères pataphysiques dans la mesure où c'est surtout dans son esprit que le cancre potache au lieu de potasser:

[...] c'est moi petit, quand je fus hors d'école, garçon buisson, toujours sorti avant quatre heures, pour se jucher, utième étage, en haut d'une table en tabouret, d'où j'extermine dans mes pensées tous les passants qui vivent en dessous. ${ }^{5}$

Plus concrètement, le flux mental toxique s'accompagne ici «d'crachats tonitruants et de parties d'bombes de poche à eau sèche», après quoi le jeune provocateur lance: «si j'étais deux au lieu d'tout seul, j'aurais gagné pour plus vous voir et très vite peint vos fesses en quatre».

\section{Potacheries et cancritude}

Devant le comportement inadmissible de ce Fantomas d'opérette, l'institution réagit mais ni réflexions («Élève Urien, seule la sortie vous intéresse $\left.{ }^{6} »\right)$, ni appréciations (mention «Pense sans les choses»), ni menaces ("Vous allez avoir un rond!») ni mauvaises notes figurant sur son bulletin patascolaire («dont trente-six d'inconduite, dix-neuf en négation, et deux en exercice d'action, dix-huit en contradiction, un quart en thème logique, quinze en falsification, et vingt sur vingt en refus ${ }^{7}$ ») n'auront aucune prise sur ce «jeune dernier», «nul en tout» et "supérieur en incapacités», qui toujours se rebiffe, «cancrant» de

3. Voir notamment le compte-rendu d'inscriptions commises par le locuteur du Discours aux animaux, op. cit., p. 57 et suiv.

4. L'Animal du temps, op. cit., 1993.

5. Le Discours aux animaux, op. cit., p. 287.

6. Ibid.

7. Je suis, I991, p. 38. 
plus belle, échouant à «l'examen des sentiments humains» et obtenant certes "très bien» en passion mais en action: "tout faux ${ }^{8}$.

Quant aux questions que l' " aigle des cancres " pose à la "vilaine maittresse" (qui «n'est pas contente» et le "[raye] d'écriture rouge»), sont-elles impertinentes ou bien jugées trop pertinentes? Quoi qu'il en soit, la sanction sera sévère, l'écolier devant apprendre une «bête listasse" pour la "saison suivante"? "Cancrer» pourra d'ailleurs être présenté comme un véritable métier puisque dans Vous qui habitez le temps, la mention «cancre à Globeval» correspond bel et bien au début d'une liste s'appliquant à des activités qui, pour certaines, semblent professionnelles (p. 4I). "Jean des Dictées", lui, semble voué à la chose scolaire (dans son cartable, il y a le rocher de Sisyphe), un peu comme si les études et l'enfance ne devaient jamais s'arrêter ou comme s'il s'agissait de "sucer du réglisse ${ }^{\mathrm{IO}}$ " jusqu'à la fin des temps. En somme, il faut pointer, aller au bureau: on évoquera d'ailleurs dans Le Discours aux animaux une "collègue d'enfance» (p. 228) ainsi qu' un "condisciple en maternelle terminale», "[mien] coscolaire en classe de creux" (p. 229) et sacripant tout autant; on y parlera également d'un "examen d'entrée en quarantième» (p. 277), d'une admission en «trente-huitième mixte» (p. 306) et même d'une «Enfance de Cent ans" (p. 82) rapprochant cet âge d'une guerre interminable. Dans L'Opérette imaginaire $^{\text {II }}$, enfin, on enviera les externes: «maintenu de force en pension, je mangeai de la pensionnade» (p. Is) - le plat n'a pas l'air ragoûtant. Pourtant, dans cet univers comiquement infernal, même l'évasion s'avère décevante et un "Jean-qui-manque-à-l'appel» en fera, tel Pinocchio, l'amer constat, lui qui fuira "l'école et ses tristes morceaux» pour des «buissons encore plus moches ${ }^{\mathrm{I} 2}$ ». Quant à la perspective de «[retourner] chez les scolaires ${ }^{13}$ », l'expression rappelle un peu «rengagez-vous, qu'y disaient!».

Punitions diverses et échecs scolaires en tous genres feront aussi l'objet d'évocations cocasses - et cela perdure au niveau de l'université avec, dans Vous qui habitez le temps, cet étudiant dont «[le] cursus fut parfait», dit-il: "sauf au finale d'examen d'art où professeur Rémy Laplace m’interroge par inadvertance sur une huile d'Osbert: "La femme à l'ananas" ; laquelle de

8. Voir Vous qui habitez le temps, I989, p. 71: «j'appris que j’avais très bien en passion mais qu'en action j'avais tout faux".

9. Dans Le Discours aux animaux, op. cit., p. 28I, le locuteur pose des questions métaphysiques à la "vilaine maîtresse» dans la marge d'un devoir: «Elle re-raya cette question d'un triple rouge zéro et me somma d'apprendre, pour la saison suivante, la bête listasse à chiffres des jours me restant encore en somme idiote de toute ma vie à parcourir."

Io. Ibid., p. 287.

II. Pièce de 1998, qui a imposé le talent comique de Novarina à l'occasion des tournées du spectacle mis en scène par Claude Buchvald.

I2. Vous qui habitez le temps, op. cit., p. 78.

13. Le Discours aux animaux, op. cit., p. 19. 
mes langues fourchant, je lus “La femme à l'ananus" " (p. 77), lapsus fatal et absolument non-rattrapable, même en deuxième session.

À l'image de l'auteur lorsqu'il était enfant ${ }^{\mathrm{T} 4}$ («refuse les sports d'équipe et joue généralement seul contre tous»), le personnage novarinien nommément désigné ("c'était justement l'air que Valère ne voulait pas chanter!») sera souvent dans une comique posture d'opposition à la norme, au groupe et à la tribu. Cela pourra s'exprimer sous la forme d'un défi a priori paradoxal comme "Tou[t] jeune, j'ai décidé d'échouer ${ }^{15}$ " ou d'un refus pur et simple du mot qu'il conviendrait d'utiliser: on n'écrira pas «fourberie» mais «foubacité»; on dira "mentardise» au lieu de "mensonge», etc. Dans La Chair de l'homme, "L'Enfant Macadémique» (et non "Académique») est encore dans une posture d'opposition (peut-être magrittienne) : «L'herbe n'est pas verte; aucune pomme n'est un fruit; le triangle est sans angles; aucun cercle n'est jamais rond ${ }^{16}$ ", et ce type de décrets comiques sera repris dans bien d'autres pièces (dans Le Jardin de reconnaissance, notamment, p. 85: "non la "douleur" mais la dadleu [...] non la "patience" mais calamon [...] non plus le "balachu" mais le tabouret», etc.).

Nonobstant, même si c'est sans doute "dans l'empêché que ça pousse», cette façon de fonctionner procède peut-être moins d'un désir violent d'anarchie que d'une incapacité fondamentale à accepter la réalité et à produire du sens à partir du langage ( «j'avais une idée par an, sans mot pour l'exprimer; trois mots par jours et sans savoir comment les attacher »). L'âge mentionné dans le Discours aux animaux ("J'étais trop petit», p. 256) n'explique pas tout; il y a un problème: «aucune idée [...] apparaissait faite comme les autres» (p. 217), «je n'avais que deux mots pour parler: sol et "ut-ré", "ut" et "tuer" » (p. 256).

En somme, on ne sait plus «à quel temps s'accrocher ${ }^{17}$ » et il ne faudrait pas croire que l'on fait exprès de les «[bouleverser $]^{18}$ », de conjuguer comme Bérurier et de ne pas suivre le cours donné: "dans ma pensée, aucune idée sortait plus vite qu'icelle émise par professeur idem» semble déplorer tel élève de Je suis paradoxalement nommé «La Grammaire» (ce qui rappelle le titre d'une pièce de Labiche); quant aux réorientations proposées à ce malheureux petit Poucet pris au piège du labyrinthe scolaire, elles ajouteront encore à sa confusion mentale et sociale et en feront même une sorte de martyr comique (mâtiné de boule de flipper) n'ayant strictement aucune prise sur son destin (p. 170):

I4. Voir G.-J. Salvy, «Vie de Valère Novarina», dans Valère Novarina, théattres du verbe, Paris, José Corti, 200I, p. 35I et suiv.

I5. Tel La Torche dans Le Drame de la vie, p. I04: "Toute jeune, j'ai décidé d'échouer.»

I6. La Chair de l'homme, 1995, p. I69.

17. Comme la Femme aux Chiffres dans Vous qui habitez le temps, op. cit., p. 67.

I8. Dans Le Discours aux animaux, op. cit., : «j’ai vécu en troublant les eaux et bouleversant les temps", p. 233. 
À Bioge, [...] au premier jour d'École de Bioge, mis déjà très tôt en apprentissage chez les mots, j'appris à être premier qui se taise puis vétéran chez des spécimens, puis trop vieux pour les minimes, d'où on me descendit immédiatement chez les petits-vétérans, où je vécus six mois en habits trop grands pour un cadet.

Enfin, un « retour à la case départ » sera préconisé: «Nous ne parvenons plus à parler avec vous qu'avec des mots que vous entendez sans savoir pourquoi. Retournez à Bioge dans ce lieu-dit dont vous n'auriez jamais dû sortir.»

La solitude qui résulte de cette forme d'impuissance semble donc accentuée par une certaine agressivité du dehors («j’appelle réel tout ce qui vient mordre») comme dans cette scène du Discours où les «inscrits du Collège réunis me voyant fuir leur société, prendre leurs maximes à contre-pied " lancent $\mathrm{au}$ "je», ici contant ses enfances, "douze quolibets» (p. 20I). Comique et douloureux, véritable "Écolier de mélancolie», ce cancre est aussi un esthète qui oppose à la dureté du monde une nouvelle conception du beau - et l'on pourra estimer que dire «L'endemain» (pour «Le lendemain») a en effet une certaine allure.

"Au commencement était la plainte» : voilà donc ce qui, au fond, explique sans doute les nombreux débordements de "L'Écolier Sacripant», son statut peu enviable de "champion de pire chez les médiocres" et le caractère violemment accusateur d'une critique acerbe, à la Ionesco (cf. La Leçon), de l'institution scolaire (épreuve du tableau noir, réorientations absurdes, scandale du bachotage, professeurs fous et/ou castrateurs, cours aberrants, etc.) ; de façon plus sous-jacente, on refusera surtout d'admettre que l'enfance, cette «mauvaise formation", soit le plus bel âge de la vie. Ajoutons par parenthèse que si les "enfances" sont dites "d'inscriptions ${ }^{19}$ ", c'est bien sûr par allusion aux écrits camouflés de l'élève Valère ${ }^{20}$ mais peut-être aussi pour dénoncer avec humour toutes les tracasseries administratives plus ou moins absurdes (fiches à remplir en début d'année, bulletins à faire signer par les parents, cahier de texte, carnet de liaison, etc.) qui, pour tout un chacun, commencent en fait dès l'école primaire.

Bref, notre «Écolier Sacripant» se réfugie dans l'art brut, l'écriture, le rire et la beauté; ce n'est pas un farceur ordinaire: il y a du Falstaff en lui. Cela posé, de qui parlons-nous au juste? D’une figure attachante et sympathique qui traverse l'œuvre toute entière, d'un personnage plus ou moins récurrent «toujours [cherchant] à qui jouer un tour», d'un «enfant fou furieux» qui

19. Le Discours aux animaux, op. cit., p. 55; les inscriptions sont un motif récurrent de cette œuvre.

20. Voir G.-J. Salvy, «Vie de Valère Novarina» sur l'année 1957, p. 353: «Rédige ses premiers écrits littéraires à la suite d'une vision éprouvée en écoutant un morceau de piano avec le casque de son poste à galène. - Dès lors, ne cesse d'écrire, mais s'en cachera jusqu'en I966.» 
(«garez-vous») «va vous éclabousser» - mais quid de l'auteur? Faut-il l'assimiler au farceur en question? Il semblerait que cela s'impose. Au fond très proche de Jean-Pierre Verheggen ${ }^{21}$, l'écrivain nous paraît en effet tout aussi insolent, blagueur, iconoclaste et démystificateur que l'écolier qu'il met en scène: c'est un peu l'«enfant terrible des Lettres françaises».

\section{Figures sacrées et autres monstres}

Au même titre que l'institution scolaire et un peu comme chez Jarry qui rapproche volontiers passion du Christ et course de côte, le sacré - par exemple et pour commencer - ne sera pas vraiment épargné par la furie potache novarinienne. Ce sera même une cible de choix: des "confessaux" seront rabelaisiennement perturbés et un prêtre « [arrosé] de germe vital» dans La Lutte des morts (p. 495) et maintes formules bibliques et liturgiques seront transformées ça et là: «Haut les louyocs, allélouliyardes», "allélouyoc», «in situ et orbi», "aux Turbies, à Orby", «Patère noustère», "Amenibus", "Que la lumière soif», "Ainsi suis-je aux lieux d'ainsi soient-ils brefs» ou encore «Saignez-vous les uns les autres» et "Homme ton prochain comme ta pomme». Dans Le Jardin de reconnaissance, Adam et Ėve, devenus un couple presque banal, regardent des émissions aussi stupides que "Je sors ma sœur" ("où il y avait un bonhomme qui arrivait à déplacer son casque avec ses yeux $\left.{ }^{22} »\right)$ et dans Le Discours aux animaux, ce sont les frères maudits de l'Ancien Testament qui se voient rapprochés de garnements infernaux comparables à Quick et Flupke: Abel «[brise] la trottinette toute neuve» de Caïn "avec [son] bras pris comme un marteau» (p. 219), lequel plus loin se venge (p. 279) en lui lançant "un os de tête de mort» qui lui revient dans la figure à la façon d'un boomerang. Jésus-Christ, lui, "Jean des trous», sera présenté comme «çui qui mit l'chapeau d'épines» - et Dieu (ce «couac») comme celui qui «passe les douanes» et qui n'est "personne en trois personnes». Nonobstant, le canular le plus drôle consistera sans doute dans ce corpus de textes miraculeusement retrouvés: "Épître aux riens», "Missive à moi et aux chiens de bois", "Lettre aux instincts», "Épître aux inhumains», "Épitaphe pour les Chiens et pour les Romains $»^{23}$.

L'histoire de la philosophie ne sera pas non plus épargnée par ce traitement de choc, le dramaturge logodynamitant potachiquement de pièce en

2I. Le poète belge J.-P. Verheggen, auteur notamment du Degré Zorro de l'écriture, Paris, Christian Bourgois, 1977, et de Ridiculum vitae, Paris, La Différence, 1994, a été un des représentants éminents de la revue TXT, qui a publié en 1975 une des premières œuvres de Novarina, Le Babil des classes dangereuses.

22. Le Jardin de reconnaissance, op. cit., p. 70. Le «Bonhomme de Terre» et la «Femme séminale" y mentionnent également Plein tarif, La Piste aux citrouilles, Rendez-moi mon moi, Dites-le deux fois deux fois, etc.

23. Formules du Chercheur de Falbala dans Vous qui habitez le temps, op. cit., p. 79. 
pièce les dialogues de Platon, se faisant tour à tour cynique ( $($ Non, non, une diminution", "Merde à l'homme!», "Il faut te gommer») et pyrrhonien ("Tout est faux», "La mort n'est pas vraie $\left.{ }^{24} »\right)$, rapprochant Rabbi Jacob de Saint Augustin $^{25}$, revisitant cocassement la pensée de Spinoza ( Bravo la viande!») et celle de Nietzsche («Ma pomme / C’est un surhomme»), réconciliant Karl et Groucho ( "Je m'évertue à plus-valoir / J'suis travaillé par l'désespoir»), hamlettisant littéralement la pensée de «Darouine» («Chimpanzé-je? [...] Ouistiti-je $\left.{ }^{26} ? »\right)$, ignorant superbement celle de Freud mais flirtant vaguement (cf. "autrui le corps») avec la phénoménologie pour finalement se reposer en compagnie de Comte-Sponville: «ça, c'est tranquille-e / J'bouffe d'la banalité en reposant sur mes pieds ${ }^{27}$ ".

Aspect peu étudié jusqu'à présent, la veine potache d'un Tim Burton - ou d'un Gotlib mêlant grandiose et saugrenu - sera également celle de notre démiurge qui propose à son tour (mais sans le revendiquer) toute une imagerie s'apparentant esthétiquement à de la SF comique, le récepteur se voyant tout à coup confronté au "Côté Farcesque de l'Abscons»: outre les inventions futuristes (thanatoscope, pulvéroscope, saucissonoscope, hallucinomètre) et les inquiétantes prophéties ("veaux en clones", "champs d'imitation", "trois bocaux de garçons et une tonne de filles, dont deux de viables", "hommes faits en autre chose que de chair d'homme»), on croisera en effet, notamment dans Le Discours aux animaux et dans Le Drame de la vie, des mutants aux pouvoirs paranormaux (voir par les oreilles, téléporter ses yeux, jeter des jets verts) s'étonnant de ce qui nous paraît aller de soi ("Qui êtes-vous, les pieds?", "D'où vient qu'on parle?»), des êtres vivants d'aspect anodin mais présentés d'une façon propre à rendre l'évidence étrange («La Femme Humaine», «chien canin », "Jean qui est», "Jean-qui-corps-a»), des monstres ayant une "bouche au front», "quatre yeux», "trois fesses», des «seins sur le dos» ou des «doigts en caoutche", un cousin d'Argus avec "douze millions d'orifices", un avatar de Grégoire Samsa doté de «six pattes en haut, six pattes en bas», une mère ayant "trois cent enfants qui tonitruent", une "femme à réaction", un certain "Jean Cerveau », un dénommé "Jean Quadrupied », "Jean Tripode»; mais aussi «L'Enfant Moteur», «L'Enfant automatique», «L'Enfant de Poche», etc. Cette

24. Derniers mots de Je suis, op. cit., p. 230.

25. Le trait d'union entre les deux personnages est bien entendu Louis de Funès, dans "Demeure fragile", un des essais du recueil Devant la parole, 1999.

26. Voir les interrogations ontologiques de L'Origine rouge, 2000, p. I03.

27. Dans L'Opérette imaginaire, 1998, L'Homme d'Outre-ça rapporte cette conversation avec sa sœur: «V'la qu'elle me lance: / «Leibniz, c'est d'la panade!»: / J'lui dis: «Sois pas toute triste / Dans ta monade.» / Puis j'fonce dans Nietzsche, à toute Bersicht / J'me dis: "Ma pomme, / C'est un surhomme!»/ Puis je rombe dans Comte-Sponville-e; / ça c'est tranquille-e / J'bouffe d'la banalité en reposant sur mes pieds!...» 
veine perdure jusque dans La Scène où un hilarant personnage multicéphale dit d'absurdes slogans politiques, et où tel autre se présente en écartant les bras pour nous "[montrer] ses autres corps». Et gageons que ces humoristiques "Chroniques du Umonde et du Urlumonde» sont loin d'être terminées.

Souvent associés, cirque et sport seront aussi concernés par le traitement humoristique: sous le chapiteau du fabuleux "Cirque Novarina», les artistes, tels Jeannot des actions comiques, Landu et Dulan, Plumo et Gomo, Régulon et Lupido, Passe-Passe, Madame Sperme, Dazy professe, le Forceur Funambul, l'homme-aquarium... s'exprimeront en effet «clouniquement» ("A l'o balustre a se balance, a va tomber, aïe-aïe») et on pourra trouver leurs numéros («l’Hélice humaine», «Féeries périlleuses», «le Jonglage Humain», «la Taupe céleste», "Capotage de la mort») comiquement pataphysiques en ce que parfaitement infaisables: "Deux jets de sperme lui sortent des poignets", "pain, viens et marche», etc. Enfin, la rhétorique sportive sera à son tour cocassement retravaillée: la triche sera dénoncée («Attention: ce mort s'est dopé»); les JO, violemment critiqués ("Crétineries Olympiques»); la figure du champion constamment moquée ("Champion du tube», "Championne du trou polisson", etc.); et l'auteur, à l'instar de Verheggen ${ }^{28}$, ira jusqu'à inventer de nouveaux sports bizarres comme «lancer du corps, plongée en confusion, foot de glas, surf ut, ping, course dans le mur et cible de la joie».

Ces précisions étant faites, il nous faut affirmer avec force que c'est surtout sur le langage lui-même que porte l'humour parodique, la "Seule à cédille» se trouvant, dans toutes ces pièces, joyeusement malmenée, qu'il s'agisse de la tradition des proverbes ("Qui me verra mort saura qu'il n’est pas moi», «Sperme en tout lieu s'efforce à plaire», «rossignol n'a pas d'parole», «Beau temps chez les morts remet tout le monde d'accord»), des structures du vocabulaire savant (hébéphronecte, paralléloperplexe, ophtalmipède, narcolyte, médiaturge, dodécatupède, Phonocrate, Philocalypse, dodécationnel ), de l'orthographe (quèque chose, finite, anéantite, voudrasse, cloudre, on direrait, siguenifie, étrumain, malaufond, nouère, oiture, oziaux) ou de la conjugaison, avec une "pluritude» de temps nouveaux : «moins que présent», "pire que passé», "presque perdu», "plus qu'attendu», "présent lointain», "futur avancé», «inactif présent", "passé postérieur", "jamais possible», "passé terminé», "possible antérieur ", «futur postérieur»... Du coup, si on en croit une didascalie de La Lutte des morts, "Les officiels sont affolés. Seuls les autres savent encore quoi dire» (p. 527) et le potache jouit de la catastrophe comique qu'il a provoquée.

28. Verheggen s'est aussi délecté de détournements sportifs et verbaux tels que "pénis de table, polo à col roulé, obèse-ball, lancer du Bengale, volley-vous coucher avec moi ». 


\section{Néologie jarryque et humour noir}

C'est aussi dans cette manière de toucher à la langue que l'auteur ressemble à Jarry: il imagine un calendrier comparable à celui du Collège ( «janure, vectiambre, marcueil, bleu, dézolet, ginindre, treptant, nolin, bucrine, élouin, soubîme, derbîme»), propose de nouvelles variations à partir du mot de Cambronne (Merdolécardi, Méjimerde, merdélessieurs) et invente à son tour des exclamations cocasses comme "Nom de jonc! ", «Gode du pape!», «Cul d'animal!» et "Joie dans les trous!». En somme, il semblerait que Novarina s'amuse à revisiter la néologie jarryque: "tuder" devient "crimer», les "oneilles» se transforment en "orilles» et la "Tempomobile» se change en "Nécromobile» (la "machine à décerveler" ne faisant qu'annoncer celle "qui dit Voici»); et quant au «bâton à merdre", au "démanche-comanche", au "crochet à nobles» et autres "petits bouts de bois dans les oneilles", ils sont remplacés par le "vide-boyau ", "l'écrasetête», le «découpe-poumon", le "pistolet-torpilleur», le «racloir énucléeur double-tranche», la "torpilleuse à pompe» et le «lame-lame à deux coups". Bref, on l'aura compris: la Chandelle Verte est loin d'être "éteindée».

Parfois, dérision rime jarryquement avec scatologie ( "J'ai porté à ce chien tout l'amour dont j'étais capable et j'en ai été récompensé au merduple») et, plus souvent, avec humour noir, que ce soit dans les inscriptions funéraires du Discours aux animaux ("Né en ", "Ci-gît méi ", "Ci-gît mon trou sans soif», "Tout est sans moi») ou dans la Chanson automobile de L'Opérette imaginaire, p. 50 et suite: "Oui vraiment! j'écrase les passants! / À chaque tournant! [...] j'remplis les poubelles / D'cyclistes à trottinettes!». À noter encore une injonction toporienne comme "Miam dans l'homme-sandwich!», des expressions comico-morbides comme "sortie par la morte», "droit comme un os", "Ho, du caveau!», «Nous sommes en vue de: Sapin», "être en os dont on fait les morts", "Demain d'accord, je change de tête de mort» et "Planches à babord! Planches à tribord!»... ou encore des noms de lieux passablement déprimants tels que "Sarcophiac», "Caveau-Plage», "place Pilori», «boulevard Nécrobiotar», "rue des Martyrs-Sociaux», «rue Jean-qui-cloche», «rue des Pessimistes» et "rue des Pompes-funèbres-de-la-mort».

Dans un registre tout aussi sombre, on tombera, dans Le Babil des classes dangereuses, qui commence par une scène de chahut où l'autorité du professeur Pinochet («Pine, tu bandes!») se voit contestée par cinq élèves particulièrement dissipés, sur une série d'inscriptions de personnages communiquant de manière moqueuse mais in fine tragique (p. 2II):

I. « Si tu te mettais à surveiller chaque seconde, au bout de peu, t'en serais fou à million» ("Le Lorrain»).

2. «Que le bouillon te tombe dessus, te lave la tête, vilain Lorrain. Signé Béhémoth du Belzébuth» ("Calineau»). 
3. «Belle pute à zob» («Le Lorrain», au canif).

4. «Deux couillons cul à cul se renvoyant la balle» («instituteur à Meximieux").

5. «Le second des sculpteurs est décédé d’une balle au front» ("Calineau»).

Autre tour pendable, l'humour de potache pouvant se faire humour de potence, ce canular morbide de la Chair de l'homme (p. I72) : "Dois-je me pendre ou pas?" J'ai envoyé cette phrase sur carte postale le $\mathrm{I}^{\text {er }}$ janvier de mes dix-huit ans à 787 correspondants relevés dans l'annuaire pour avoir porté le nom de Corde ou Lacorde. » Avec des patronymes évoquant la ficelle, l'effet est moins réussi. Quant aux réponses, elles ne seront pas moins drôles: "Pendstoi oui si ça te chante», "Pends-toi oui mais pends-toi vite», "Pends-toi ou te pends pas mais décide-toi ", "Ne te pends pas, mais ne te plains pas plus tard de t'être pas pendu.»

Ce type de blague étant possible, on se prend à penser qu'on est en présence de faits d'armes véritables; or, si l'on se réfere à la mini-biographie publiée chez Corti, on se rend compte que la "cancritude comique» était effectivement un peu le propre de l'enfant que fut Valère Novarina ${ }^{29}$ :

I95I

[...] Premier bulletin scolaire: "Falsifie ses notes et gribouille mes observations."

I955

[...] Est mis dans la rangée des cancres, et l'institutrice, qui s'est fait teindre les cheveux en bleu, refuse de le présenter à l'examen d'entrée en sixième. Il s'y présente seul et sera reçu premier.

[Une autre année] :

Prix de français. / Reçu dernier sur trois cents candidats au BEPC.

À l'image de la maîtresse aux cheveux bleus, c'est peut-être le récepteur de l'œuvre lui-même qui est devenu la cible privilégiée de l'écrivain farceur; de fait, s'il ne boxe pas dans la même catégorie artistique que le redoutable Entarteur, il semblerait qu'il lance lui aussi, Novarina, de véritables tartes à la crème, symboliques celles-là, théâtrales, littéraires, poétiques, pataphysiques, verheggeniennes à la face de certaines institutions en place, ceci - sans doute en partie par altruisme et agacement - dans le but de dénoncer avec force et violence des réalités effectivement de plus en plus insupportables comme l'aliénation par le travail, les guerres absurdes, la druckérisation de l'esprit, le décervelage, le triomphe de Boucot en ce début de troisième millénaire.

L'anarchie en question est d'ailleurs parfois moins symbolique qu'il n'y paraît: "pour me venger de mon patron, j'ai mal vissé tous les boulons ${ }^{30}$ »:

29. G.-J. Salvy, «Vie de Valère Novarina», op. cit., p. 352 à 354.

30. La Chair de l'homme, op. cit., p. 59. 
s'agirait-il de perturber chaplinesquement le fonctionnement inhumain de quelque "Atelier violent»? De donner vraiment la parole à Charlot? De parler au nom des opprimés, des empêchés, des cancres et du dernier de la classe? Cela n'est pas impossible.

Dans des pièces comme L'Origine rouge et La Scène, c'est sur la «machine à dire Voici » que Novarina choisira de tirer à boulets rouges - et notamment sur le sacro-saint " $\mathrm{JT}^{3 \mathrm{I}}$ ", avec des nouvelles absurdes, terrifiantes et drôles rappelant tout à la fois Sade (celui des I20 journées), Détective, Coluche et les Inconnus: «les Anthropopantropes Lumnotophes ont malheureusement éventré tous les partisans d'Elomire Barnophle», "les énuclées se comptent désormais malheureusement hélas par dizaines de milliers »; "à l'occasion des fêtes annuelles de la Bobancrasserie, plusieurs Mam’loubouchi se sont tranché la gorge puis pelés au couteau»; ou encore: "Le forcené d'Oléron vient de mettre fin à ses jours. Voyez les corps des cent quarante petites victimes allongées dans l'ordre. Dans le sang encore frais, Marie-Odile Bouchoux». Quant aux jeux ("Où est né Lecanuet? Faux. Tu es collé, retourne à ton clapier»), ils ne seront pas oubliés, de même que certains autres programmes tout aussi abêtissants comme cette soirée au cours de laquelle «nous verrons le ministre de l'Agriculture traire une vache» et telle émission de débat qui "réunira le panel des autorités compétentes sur le thème "La pédophilie est-elle un mal nécessaire? " "; dans une troisième, certains VIP "accepteront ou pas le défi de venir devant nos caméras - faire leurs besoins».

Précisons pour terminer que ce réjouissant jeu de massacre ne concernera certainement pas que le petit écran, l'auteur s'amusant également à parodier sondages («I pour cent éprouve couvercle», «O,O2 pour cent mangent onze biscottes») et horoscopes («Heureux les Béliers! Taureaux : les horloges! Balances: gare à vous. Et attention les Lions! Sagittaires: attention aux lions. Tout beaux, les Verseaux!»), formules administratives ("vous présentant, Monsieur le directeur, l'expression de mes sentiments colorés») et slogans publicitaires ( «Suivez Ci-Gît!», «Mangez des ours!», «Dormez de marbre!», «changez de viande!», «pétez de soie!», «mourrez sobre!», «datez du jour!»). Il se moquera de la politique ${ }^{32}$ («Votez utile, votez Martine Filleul!», «Paul Pendu : le choix de se taire», "Non merci la déconfiture», «Maintenant ça va changer», "Osons la Bretagne pour changer la République», «Bruno Canet: La passion d'en découdre»), fustigera la culture d'entreprise (à coups de prosperiting, vitaliting, nervositing, impondérability, fatiguing, abrutising, conjoncturing-

3I. Voir notamment les informations internationales des deux "Machines à dire voici» dans L'Origine rouge, op. cit., p. 45 et suiv.

32. En particulier dans le grand débat d'opinions de La Scène, p. I2I à I27, conclu par Diogène déclarant que «Le peuple souverain passe de cour à jardin.» 
technicising-industriality), dénoncera la mascarade humanitaire ("Médecins Sans Scrupules", "Profits sans frontières») et critiquera moliéresquement l'institution médicale ( "Je vous préviens, j'en ai assassiné plus d'un»).

Ici, esprit d'enfance, humour noir, gros rire et comique canularesque semblent fonctionner comme une réponse potache, un pied de nez pataphysique à un monde perçu comme truqué, faussement sérieux et soi-disant adulte et à un réel social ressenti comme agressif, brimant, scolaire et trop normatif.

Autre aspect pointé par de nombreux critiques, il semblerait qu'il y ait chez Novarina (proche en cela de Beckett, Queneau, Ghelderode, Duchamp, Dubuffet, Tardieu, Topor, Pinget ou Dubillard) une certaine sophistication, un certain raffinement mais allant toujours de pair avec des éléments burlesques, grotesques, ludiques et/ou incongrus voire grossiers; ce type de dualité se retrouve également chez son "frère d'armes", Jean-Pierre Verheggen, qui comme lui écrivit sur Artaud et dont le mirlitonisme assumé nous paraît relever de la plus haute poésie - affirmons-le pour adresser in fine un clin d'œil au «Falstaff belge»: il y a du Verheggen chez Novarina (et pas seulement en matière de comique et de "potachité»).

"Écolier Sacripant», "enfant mirliton», "Garçon buisson», "Cancre logique», "jeune dernier»: autant de dénominations cocasses pouvant donc s'appliquer à celui qui dit "je» dans les pièces de Valère Novarina: «[il] y a en nous un esprit joueur» suggère l'auteur - à nous de le retrouver au lieu de jouer au Docteur: tel nous semble être un des seuls messages à peu près compréhensibles de cette œuvre si bellement hermétique. 